\title{
Comfortable dental masks
}

Kent Express has introduced a new range of surgical masks from its own Kent Dental range. Kent Dental Earloop Procedure Masks are comfortably fitting

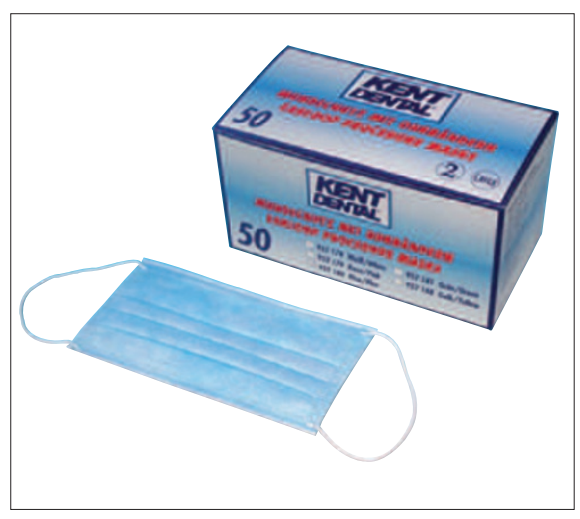

\section{Increased focal range}

Schick Technologies has launched its next generation of intraoral camera the Schick USBCam2. It has an increased focal range so you can capture images from extreme close-up to full arch without having to adjust the focus.

There are eight LED lights which give extra bright illumination for optimum image clarity and colour reproduction and for ease of use the one-click image dental masks incorporating earloops.

The masks are soft, light, latex-free and ensure no plastic will ever touch the wearer's skin. With non-irritant inner and outer facing surrounds, glass-free filter media and a full width nosepiece, a snug, secure and odourless fit is assured. The masks feature high bacterial filtration efficiency and offer low breathing resistance. Kent Dental also offers Pleated Tie-On masks with the additional benefit of a cloth-like tie.

Both masks are non-irritating and available in a range of colours. The Earloop Procedure Masks are presented in white, pink, blue, green or yellow, and Tie-On masks in green, blue and white. Reader response number 50

capture button is conveniently located on the rear of the camera.

The new camera joins the current model, the Schick USBCam which is also offered, and both are "plug and play' as each connects to any PC with a standard USB port and is ready for use. A separate video capture card is not required. Both cameras also have their own internal power source giving you complete mobility.

Reader response number 51

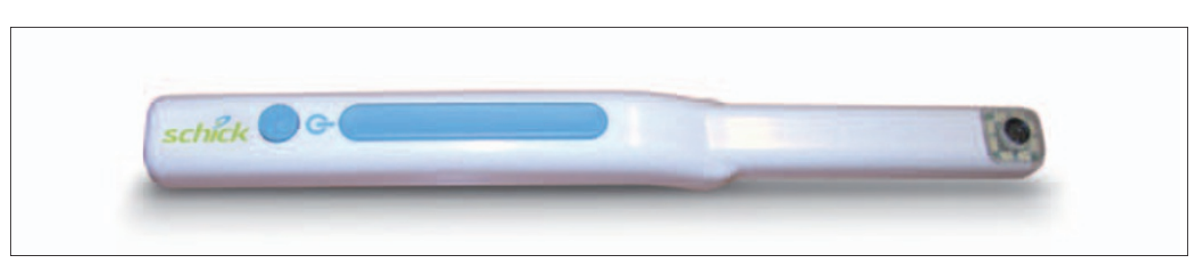

\section{High levels of infection control}

Metasys's GreenctClean range of hygiene products provides you with the highest possible level of infection control. With numerous products within the range, you will find the solution that you are looking for whether it's alcohol-free that is suitable for delicate surfaces or highly concentrated cost saving products.

All disinfectants from Metasys are biodegradable, helping to look after the planet and each disinfectant has a CE sign. Metasys supply a range of information posters that can be hung up in the practice and can be particularly useful as a reminder to staff.

Reader response number 52

\section{Impressions without bubbles}

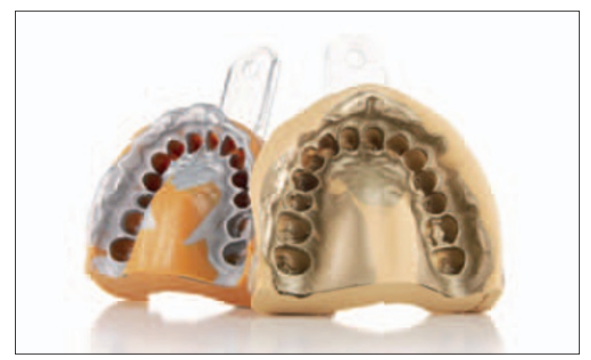

Coltène/Whaledent has launched Affinis Precious, the next generation of wash materials, which has been further developed in three specific areas. It shows spontaneous flow behaviour after application, particularly on wet surfaces. In addition, in spite of the good flow properties the material still retains its shape and does not drip when in place. This immediate and sustained surface activation (hydrophilia) makes it possible to achieve detailed impression results without bubbles and voids.

The special matt silver and matt gold precious metal shades of Affinis Precious in combination with all Affinis tray materials provide ideal contrast and optimise the detail readability. This makes it quick and easy to assess the impression result.

It has a clinical processing time of up to 60 seconds and the oral setting time is 120 seconds. All Affinis tray materials have the same oral setting time for faster and more accurate impressions. A fastbinding type is unnecessary.

Reader response number 53 


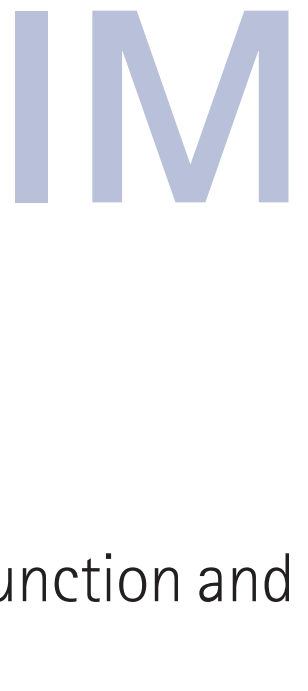

'Quality of life' is an important factor for edentulous patients who are looking for ways to improve the function and aesthetics of dentures and it has subsequently become more important for dentists to offer treatments that will help overcome their worries.

Straumann Implants offer major advantages over conventional dentures by providing a stable foundation and helping preserve the adjacent teeth and bone structure. Dentures can then be
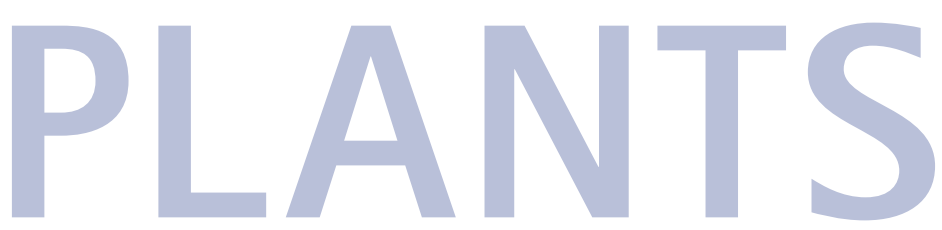

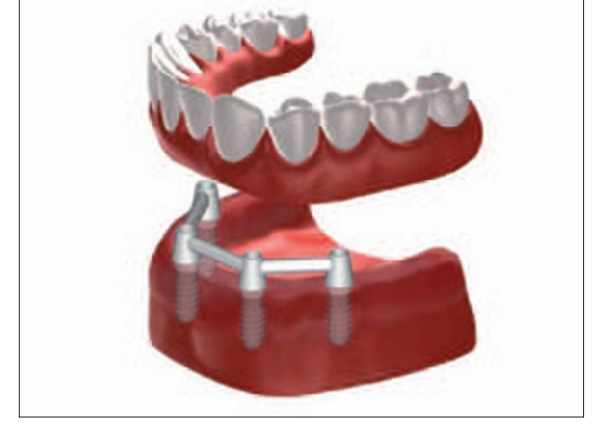

attached to an anchorage and can be easily removed for cleaning and replaced with a simple, firm fit.

Reader response number 54

\section{Versatile implant system}

Osteotomes from Astra Tech have been specifically designed to match and compliment Astra Tech's versatile implant system. The Astra Tech OsseoSpeed implant installed with the Osteotome technique provides improved conditions when bone quality and quantity are compromised.

Osteotomes are ideal for ridge expansion, bone condensing, sinus floor elevation procedures and for both one-stage and two-stage surgery.

Reader response number 55 


\section{Predictable crestal bone preservation}

The Certain Prevail Implant from 3i Implant Innovations has been designed to increase the potential for more predictable crestal bone preservation. The design features Integrated Platform Switching which provides the marketplace with a simple and predictable option for potentially achieving better crestal bone preservation, which would result in optimal soft tissue aesthetics.

The implant incorporates a coronal bevel design that medialises the implantto-abutment junction.

Reader response number 56

\section{Ultrasonic implant scaler}

Dentsply has launched a new magnetostrictive, ultrasonic implant scaler. The Cavitron SofTip implant insert means there is now no need to switch from ultrasonic scaling to hand instruments when faced with an implant patient.

It is imperative that the abutment and surrounding structures of an implant are free from calculus, deposits and biofilm for proper osseointegration. Most dental implants fail due to the build-up of mineralised deposits and the presence of infection in the surrounding tissues causing peri-implantitis.

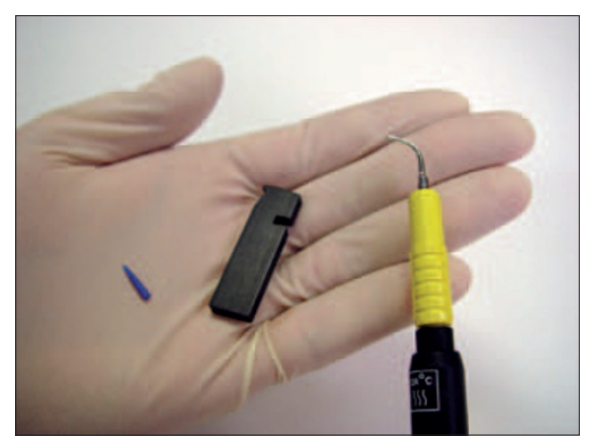

The Cavitron SofTip Implant insert helps remove calculus and deposits thus aiding in the implementation of the implant, for improved patient oral health and a successful implant.

Reader response number 57

\section{High visibility}

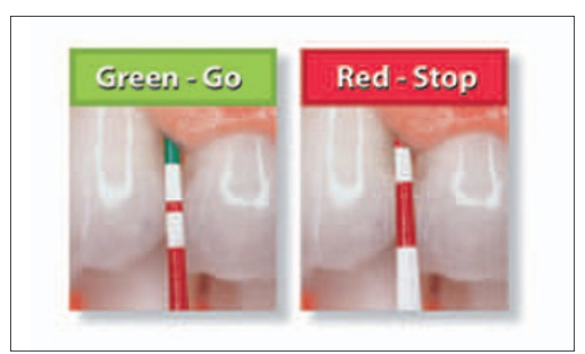

The Periowise multi-coloured periodontal probe is easy to read, gentle on tissue and safe to use around implant fixtures. Clear, well-defined markings in green and red give high visibility against tooth substance and soft tissue and ensure accurate pocket depth readings.

A choice of markings provide for individual preferences and the flexible tip gives good tactile sense and reduces the risk of tissue trauma or damage to implant fixtures. Made from an autoclavable polymeric material which can also be ultrasonically cleaned, PerioWise eliminates the most frustrating part of periodontal probing: poor readability. At a glance, you can quickly determine the depth of the pocket and make the appropriate diagnosis.

Periowise is supplied in packs of three or six and is available from all major dental dealers.

Reader response number 58 